\title{
Physical and Electrochemical Properties of Monofluorinated Ethyl Acetates for Lithium Rechargeable Batteries
}

\author{
Noritoshi Nanbu, ${ }^{\mathrm{a}, *}$ Yuta Suzuki, ${ }^{a}$ Kiyoshi Ohtsuki, ${ }^{\mathrm{a}}$ Takeshi Meguro, ${ }^{\mathrm{a}}$ \\ Masahiro TAKeHARA, ${ }^{\mathrm{b}}$ Makoto UE, ${ }^{\mathrm{b}}$ and Yukio SASAKI ${ }^{\mathrm{a}}$ \\ aDepartment of Life Science and Sustainable Chemistry (Former name: Department of Nanochemistry), Faculty of \\ Engineering, Tokyo Polytechnic University (1583 Iiyama, Atsugi, Kanagawa 243-0297, Japan) \\ bMitsubishi Chemical Group Science and Technology Research Center, Inc. (8-3-1 Chuo, Ami, Inashiki, Ibaraki 300- \\ 0332, Japan)
}

Received December 9, 2009 ; Accepted January 8, 2010

\begin{abstract}
Ethyl acetate (EA) shows low viscosity for its relative permittivity. Monofluorinated solvents exert the polar effect on the physical and electrochemical properties. Relative permittivity of 2-fluoroethyl acetate (2FEA) was lower than that of ethyl fluoroacetate (EFA), though viscosity of $2 \mathrm{FEA}$ was higher. Ionic conductivity of $2 \mathrm{FEA}$ was lower than that of EFA, but higher than that of EA at high temperatures. The anodic stability of $2 \mathrm{FEA}$ and EFA was higher than that of EA. The use of 2FEA as a co-solvent improved cycling efficiency of a lithium anode.
\end{abstract}

Key Words : Monofluorinated Solvent, Carboxylate, Position Isomerism, Lithium Battery

\section{Introduction}

Ethyl acetate (EA) is classified as a carboxylate. EA shows high relative permittivity $\left(6.02\right.$ at $\left.25^{\circ} \mathrm{C}\right),{ }^{1)}$ but low viscosity $\left(0.426 \mathrm{mPa} s\right.$ at $\left.25^{\circ} \mathrm{C}\right),{ }^{1)}$ as compared to dimethyl carbonate (DMC) $\left(\varepsilon_{\mathrm{r}}=3.12\right.$ and $\eta=0.59 \mathrm{mPa} \mathrm{s}$ at $\left.25^{\circ} \mathrm{C}\right)$, ethyl methyl carbonate $(\mathrm{EMC})\left(\varepsilon_{\mathrm{r}}=2.93\right.$ and $\eta=0.65 \mathrm{mPa} \mathrm{s}$ at $25^{\circ} \mathrm{C}$ ), and diethyl carbonate (DEC) $\left(\varepsilon_{\mathrm{r}}=2.82\right.$ and $\eta=0.75 \mathrm{mPa} \mathrm{s}$ at $\left.25^{\circ} \mathrm{C}\right) .{ }^{1)} \mathrm{DMC}$, EMC, and DEC are commonly used as low-viscosity solvents for lithium-ion batteries. ${ }^{2,3}$ The use of carboxylates as alternative solvents can decrease the internal resistance of lithium batteries. However, the oxidative-decomposition potentials of carboxylates $(3.4 \mathrm{~V}$ vs. SCE for methyl acetate and methyl propionate $)^{4)}$ are lower than those of chain carbonates (3.7 V vs. SCE for DMC, EMC, and DEC). ${ }^{4)}$ Partially fluorinated cyclic and chain carbonates exert the polar effect on the physical and the electrochemical properties such as permittivity, viscosity, ionic conductivity, and electrochemical stability. ${ }^{5-8)}$ Monofluorination of carboxylates can also improve the anodic stability. Methyl difluoroacetate is known as an excellent additive for lithium batteries. ${ }^{2,9-13)}$

In this paper, we describe the effect of position isomerism on the physical and electrochemical properties of two monofluorinated EAs: 2-fluoroethyl acetate (2FEA) and ethyl fluoroacetate (EFA). Figure 1 shows the struc-<smiles>CC(=O)OCCF</smiles><smiles>CCOC(=O)F</smiles><smiles>CCOC(C)=O</smiles><smiles>CCOC(=O)OC</smiles> 
tures of 2FEA, EFA, EA, and EMC.

\section{Experimental}

We synthesized 2FEA from acetyl chloride and 2-fluoroethanol in the presence of pyridine. 2FEA was prepared in a yield of about $60 \%$. After removal of pyridine by adding dilute $\mathrm{HCl}$ solution, the product was collected and was purified under reduced pressure first by simple distillation and then by fractional distillation. The purity of $2 \mathrm{FEA}$ was determined to be more than $99.9 \%$ by means of gas chromatography equipped with a FID detector (Shimadzu Corp., GC-1700). 2FEA was dehydrated with purified molecular sieves $4 \mathrm{~A}$ before use. 2Fluoroethanol was obtained by distillation of a mixture of ethylene carbonate (EC) and potassium hydrogendifluoride $\left(\mathrm{KHF}_{2}\right)$ after heated in 1-methyl-2-pyrrolidinone (NMP). EC, EMC, EA, and $\mathrm{LiPF}_{6}$ were used as received (Kishida Chemical Co., Ltd., LBG grade for EC, EMC, and EA; Stella Chemifa Corp. for $\mathrm{LiPF}_{6}$ ).

The apparatus and techniques for measurements are essentially the same as those previously reported.,8)

\section{Results and Discussion}

\section{1 Physical properties}

Figure 2 shows the temperature ( $\theta$ or $T$ ) dependence of (a) relative permittivity $\left(\varepsilon_{\mathrm{r}}\right)$ and (b) viscosity $(\eta)$ of $2 \mathrm{FEA}, \mathrm{EFA}$, and EA single solvents. In addition, this figure compares data for EMC, whose structure is similar to that of EA. The relative permittivity reflects the ease of dielectric polarization. The relative permittivity has a significant effect on the strength of the interactions between ions in an electrolyte solution. The viscosity is regarded as an internal friction based on intermolecular forces and affects the ionic conductivity. Relative permittivity of EA was more than twice that of EMC in spite of its low viscosity. Monofluorination of EA increased both the relative permittivity and the viscosity. Interestingly, the relative permittivity of EFA was higher than that of
2FEA, though the viscosity of EFA was lower. An acetyl group $\left(\mathrm{CH}_{3} \mathrm{CO}-\right)$ is more electron-withdrawing than an ethoxy group $\left(\mathrm{CH}_{3} \mathrm{CH}_{2} \mathrm{O}-\right)$. Monofluorination of the acetyl group can make the opposite large charges separated in the solvent molecule, and consequently induce the large bond moment. On the other hand, monofluorination of the ethoxy group can greatly enhance the internal friction. The monofluorinated ethoxy group rotates freely and has spread spatially as compared to the monofluorinated acetyl group. The individual bond moments are partly canceled out in the 2FEA molecule. Thus, an EFA molecule has a net dipole moment that is larger than that of a $2 \mathrm{FEA}$ molecule. The intermolecular hydrogen bonding $(\mathrm{CF}-\mathrm{H} \cdots \mathrm{O}=\mathrm{C}$ or $\mathrm{C}-\mathrm{H} \cdots \mathrm{F}-\mathrm{C})$ is also responsible for the cooperative orientation of the EFA molecules. The increased viscosity of 2FEA is supported by the finding that the molar concentration of 2FEA was slightly higher than that of EFA molecules in spite of the same molar mass: $10.31 \mathrm{~mol} \mathrm{dm}^{-3}$ for 2FEA, $10.23 \mathrm{~mol}$ $\mathrm{dm}^{-3}$ for EFA, $10.15 \mathrm{~mol} \mathrm{dm}^{-3}$ for EA, and $9.70 \mathrm{~mol} \mathrm{dm}^{-3}$ for EMC at $25^{\circ} \mathrm{C}$. The molar concentration $(c)$ is calculated from density $(d)$ and molar mass $(M)$ according to the following equation: $c=d / \mathrm{M}$.

The viscosity decreased exponentially with an increase in temperature, and plots of $\log (\eta / \mathrm{mPa} s)$ vs. $T^{-1}$ gave straight lines. The high translational kinetic energy allows intermolecular attractions to be overcome more easily, and the internal friction is reduced at high temperatures. The apparent activation energy for viscosity $\left(E_{\mathrm{a}, \eta}\right)$ is obtained from the relation proposed by Andrade: $\eta=A_{\eta} \exp \left(E_{\mathrm{a}, \eta} / R T\right){ }^{14)}$ The apparent activation energy is determined to be $12.41,12.02,11.13$, and $8.258 \mathrm{~kJ} \mathrm{~mol}^{-1}$ for 2FEA, EFA, EMC, and EA, respectively. The viscosity and apparent activation energy of monofluorinated EAs were higher than those of EA and EMC. The apparent activation energy decreased in the same order as the viscosity: $2 \mathrm{FEA}>\mathrm{EFA}>\mathrm{EMC}>\mathrm{EA}$.

\section{$T / \mathrm{K}$}
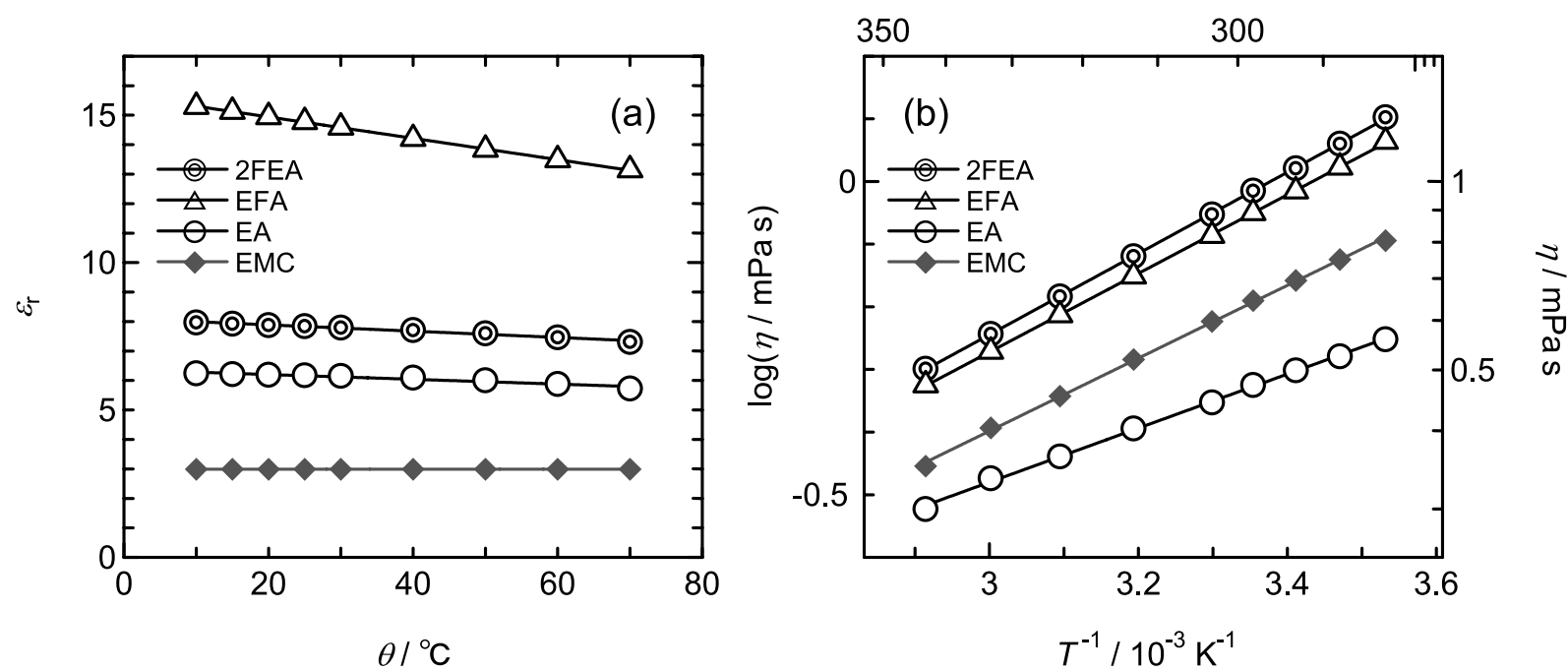

Fig. 2 (a) Relative permittivities $\left(\varepsilon_{\mathrm{r}}\right)$ and $(\mathrm{b})$ viscosities $(\eta)$ of $2 \mathrm{FEA}$, EFA, EA, and EMC single solvents as a function of temperature $(\theta$ or $T)$ from $10{ }^{\circ} \mathrm{C}(283.15 \mathrm{~K})$ to $70{ }^{\circ} \mathrm{C}(343.15 \mathrm{~K})$. 


\section{2 Ionic conductivity}

Conductivity of an electrolyte solution is a key factor determining the internal resistance and rate performance of a battery. Figure 3 shows the temperature $(\theta)$ dependence of conductivities $(\kappa)$ of $2 \mathrm{FEA}, \mathrm{EFA}, \mathrm{EA}$, and EMC single solutions containing $1 \mathrm{~mol} \mathrm{dm}^{-3} \mathrm{LiPF}_{6}$. The conductivities of the electrolyte solutions increased with an increase in temperature. This is because viscosities of the solvents decrease rapidly at high temperatures, as shown in Fig. 2(b). The decrease in relative permittivity at high temperatures would not affect the ionic conductivity so much. The conductivity can be affected by the ionic mobility, the charge numbers of the ions, the concentration of the electrolyte, the degree of ionic dissociation, etc. The ionic mobility relates to viscosity of the

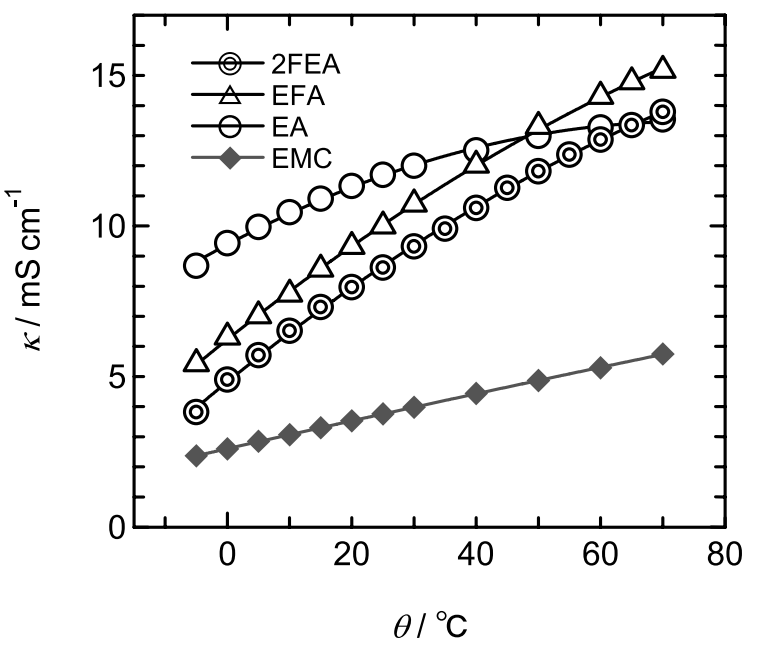

Fig. 3 Conductivities $(\kappa)$ of $2 \mathrm{FEA}, \mathrm{EFA}, \mathrm{EA}$, and EMC single solutions as a function of temperature $(\theta)$ from $-5{ }^{\circ} \mathrm{C}$ to $70{ }^{\circ} \mathrm{C}$. Electrolyte: $\mathrm{LiPF}_{6}\left(1 \mathrm{~mol} \mathrm{dm}{ }^{-3}\right)$. electrolyte solution, while the degree of ionic dissociation of the lithium salt is set by the balance between the permittivity and Lewis basicity of the medium. ${ }^{8)}$ The conductivity of an EA solution was more than twice that of an EMC counterpart. Despite its high relative permittivity, the viscosity of EFA was lower than that of $2 \mathrm{FEA}$, as described in the previous section. Therefore, the high degree of ionic dissociation and the high ionic mobility results in the synergistic effect on the increased conductivity of the EFA solution: The conductivity of an EFA solution was higher than that of a $2 \mathrm{FEA}$ counterpart over the temperature range investigated. The introduction of a fluorine atom into an EA molecule may decrease the electron-pair donability of oxygen atoms in the -COO- moiety. This effect results in the decreased solvation to lithium ion and, consequently, in the lower degree of ionic dissociation. In particular, the monofluorination of the alkoxy group may decrease Lewis basicity more greatly than that of the acetyl group.

There was intersection of the two plots of conductivity against temperature: about $65{ }^{\circ} \mathrm{C}$ for $2 \mathrm{FEA}$ and EA; and about $47^{\circ} \mathrm{C}$ for $\mathrm{EFA}$ and $\mathrm{EA}$. The temperature range where the monofluorination evoked the increase in the conductivity was wider for EFA and EA solutions than for 2FEA and EA solutions. EFA shows low viscosity at high temperatures but maintains moderate permittivity and Lewis basicity. The threshold temperature in the conductivity is also observed for 2-fluoroethyl methyl carbonate (2FEMC) and EMC solutions (about $\left.45{ }^{\circ} \mathrm{C}\right) ; ;^{15}$ and ethyl 2-fluoroethyl carbonate (E2FEC) and DEC solutions (about $25{ }^{\circ} \mathrm{C}$ ) at $1 \mathrm{~mol} \mathrm{dm}^{-3}$ of $\mathrm{LiPF}_{6}{ }^{16}$ )

\section{3 Electrochemical stability and cycling efficiency of} lithium anode

Linear sweep voltammetry (LSV) was carried out to investigate electrochemical potential windows of electrolyte solutions. Figure 4(a) shows linear sweep voltam-
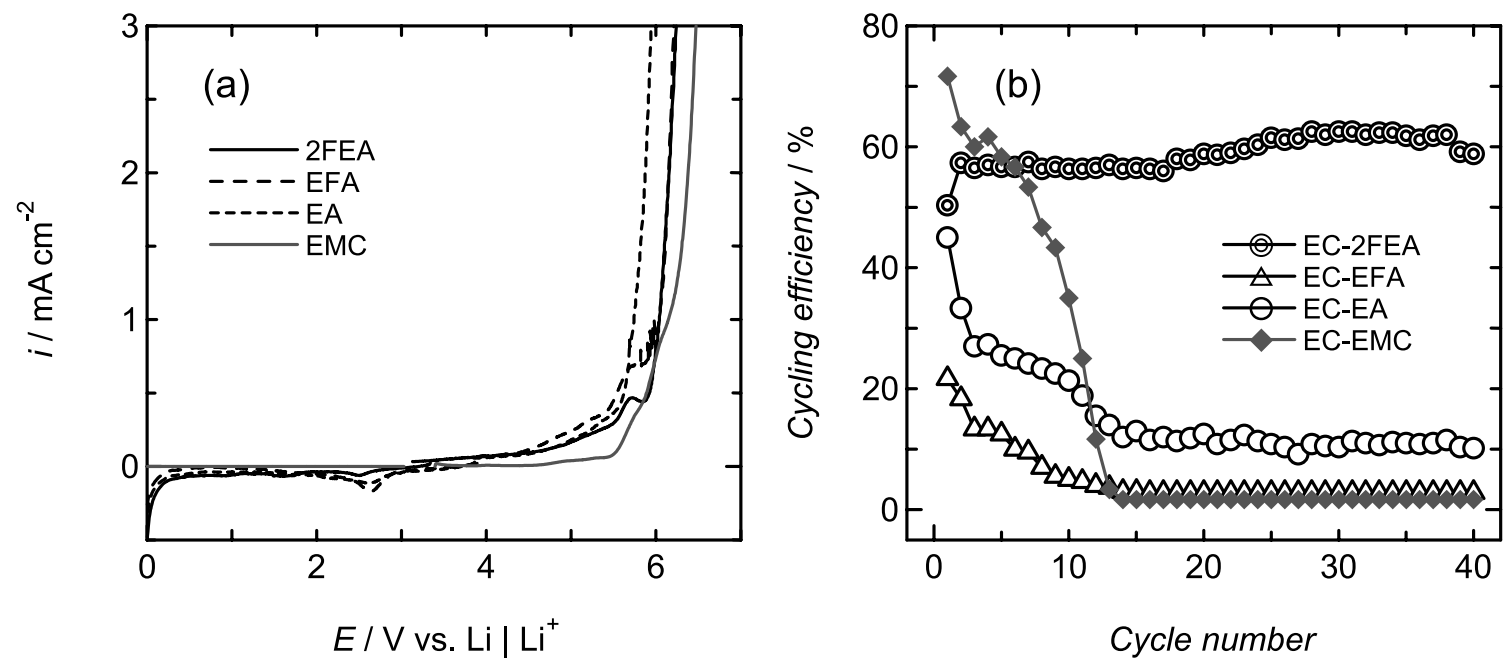

Fig. 4 (a) Linear sweep voltammograms obtained with a platinum electrode for 2FEA, EFA, EA, and EMC single solutions at a scan rate of $5 \mathrm{mV} \mathrm{s}^{-1}$ at $25^{\circ} \mathrm{C}$. These solutions contain 1 mol dm ${ }^{-3} \mathrm{LiPF}_{6}$. (b) Variation of cycling efficiency of a lithium anode with respect to the cycle number at $25{ }^{\circ} \mathrm{C}$. Electrolyte solutions: EC-2FEA, EC-EFA, EC-EA, and EC-EMC equimolar binary solutions containing $1 \mathrm{~mol} \mathrm{dm}^{-3} \mathrm{LiPF}_{6}$. The plating and the stripping current density were fixed at $\pm 1 \mathrm{~mA} \mathrm{~cm}^{-2}$. The plated charge density was $-300 \mathrm{mC} \mathrm{cm}{ }^{-2}$, and the cut-off voltage was set at $+1.0 \mathrm{~V} \mathrm{vs}$. $\mathrm{Li} \mid \mathrm{Li}^{+}$during the anodic stripping process. 
mograms obtained with a platinum electrode for 2FEA, EFA, EA, and EMC single solutions at a scan rate of 5 $\mathrm{mV} \mathrm{s}{ }^{-1}$ at $25{ }^{\circ} \mathrm{C}$. These solutions contain $1 \mathrm{~mol} \mathrm{dm}^{-3} \mathrm{LiPF}_{6}$ as a single electrolyte. Monofluorination of EA increased the anodic stability, as easily predicted by the highest electronegativity of fluorine. The anodic stability of 2FEA and EFA was slightly lower than that of EMC. Unlike anodic decomposition, a small current flowed in a potential range of 3 to $0 \mathrm{~V}$. We cannot determine the exact cathodic-decomposition potentials of $2 \mathrm{FEA}$, EFA, EA, and EMC. Small peaks observed at about $5.8 \mathrm{~V}$ can be ascribed to degradation of trace amounts of $\mathrm{LiPF}_{6}$ or impurities in 2FEA and EFA.

Cycling efficiency of a lithium anode indicates coulometric efficiency achieved with the repetitiveness of the deposition and dissolution of lithium on a nickel electrode. Figure 4(b) shows the variation of the cycling efficiency of a lithium anode with respect to the cycle number at $25^{\circ} \mathrm{C}$. We used EC-2FEA, EC-EFA, and EC-EA equimolar binary solutions containing $1 \mathrm{~mol} \mathrm{dm}^{-3} \mathrm{LiPF}_{6}$. This figure compares data for an EC-EMC equimolar binary solution. The cycling efficiency was less than $20 \%$ for EC-EFA, EC-EA, and EC-EMC binary solutions after the 12th cycle. The use of the EC-2FEA binary solution remarkably suppressed the cycling efficiency fading. The cathodic decomposition of the EC-2FEA binary solution on a lithium anode can form a passive film containing proper amounts of fluorine compounds. Although the fluorine compounds in the surface film have not been identified, they would show moderately low electron-pair donicity. The large amount of lithium ion can become incorporated into the surface film, and the lithium ion may readily pass through the surface film. The morphology, thickness, density, and chemical composition of the surface film may be different from those of a surface film formed in a lithium anode|ECEFA solution interphase. The possible organofluorine compounds in the surface films are $\mathrm{CH}_{2} \mathrm{FCH}_{2} \mathrm{OLi}$ and $\mathrm{CH}_{2} \mathrm{FCOOLi}$ in 2FEA- and EFA-based systems, respectively.

\section{Conclusion}

We have investigated the effect of position isomerism on the physical and electrochemical properties of two monofluorinated carboxylates: 2FEA and EFA. Although viscosity of EFA was lower, relative permittivity of EFA was higher than that of 2FEA. Consequently, ionic conductivity of EFA was higher than that of 2FEA. The ionic conductivity of 2FEA was higher than that of EA at high temperatures. The anodic stability of $2 \mathrm{FEA}$ and EFA was higher than that of EA. The use of an EC2FEA equimolar binary solution remarkably suppressed the cycling efficiency fading.

\section{References}

1) J. A. Riddick, W. B. Bunger, and T. K. Sakano, Organic Solvents: physical properties and methods of purification, 4th edn., Wiley-Interscience, New York (1986).

2) J. Yamaki, Advances in Lithium-Ion Batteries (Eds. W. A. van Schalkwijk and B. Scrosati), Kluwer Academic / Plenum Publishers, New York, Chap. 5 (2002).

3) D. Aurbach and A. Schechter, Lithium Batteries, Science and Technology (Eds. G.-A. Nazri and G. Pistoia), Kluwer Academic Publishers, Boston, Chap. 18 (2004).

4) M. Ue, Richiumu-ion Niji-denchi, Zairyo to Oyo, 2nd edn. (Eds. M. Yoshio and A. Kozawa), The Nikkan Kyogyo Shimbun, Ltd., p. 84 (2000) [in Japanese].

5) N. Nanbu, M. Takehara, S. Watanabe, M. Ue, and Y. Sasaki, Bull. Chem. Soc. Jpn., 80, 1302 (2007).

6) N. Nanbu, K. Takimoto, K. Suzuki, M. Ohtake, K. Hagiyama, M. Takehara, M. Ue, and Y. Sasaki, Chem. Lett., 37, 476 (2008).

7) N. Nanbu, K. Takimoto, M. Takehara, M. Ue, and Y. Sasaki, Electrochem. Commun., 10, 783 (2008).

8) N. Nanbu, S. Watanabe, M. Takehara, M. Ue, and Y. Sasaki, J. Electroanal. Chem., 625, 7 (2009).

9) T. Nakajima, K. Dan, M. Koh, T. Ito, and T. Shimizu, J. Fluorine Chem., 111, 167 (2001).

10) J. Yamaki, I. Yamazaki, M. Egashira, and S. Okada, J. Power Sources, 102, 288 (2001).

11) K. Sato, I. Yamazaki, S. Okada, and J. Yamaki, Solid State Ionics, 148, 463 (2002).

12) M. Ihara, B. T. Hang, K. Sato, M. Egashira, S. Okada, and J. Yamaki, J. Electrochem. Soc., 150, A1476 (2003).

13) T. Kawamura, T. Tanaka, M. Egashira, I. Watanebe, S. Okada, and J. Yamaki, Electrochem. Solid-State Lett., 8, A459 (2005).

14) E. N. Andrade, Nature, 125, 309 (1930).

15) M. Takehara, N. Tsukimori, N. Nanbu, M. Ue, and Y. Sasaki, Electrochemistry, 71, 1201 (2003).

16) N. Tsukimori, N. Nanbu, M. Takehara, M. Ue, and Y. Sasaki, Chem. Lett., 37, 368 (2008). 